\title{
Surgical treatment for acute rupture of iliac artery aneurysm caused by invasion of retroperitoneal leiomyosarcoma
}

\begin{abstract}
Primary retroperitoneal leiomyosarcoma and isolated iliac artery aneurysms are both rare diseases. This article reports a case of primary pelvic retroperitoneal leiomyosarcoma in which the tumor invaded the right common iliac artery aneurysm causing arterial wall erosion, and aneurysmal rupture resulting in intra-abdominal hemorrhage. We successfully resected the retroperitoneal leiomyosarcoma and repaired the iliac artery aneurysm by artificial vascular transplantation. No postoperative complications occurred.

Primary retroperitoneal leiomyosarcoma is a rare soft tissue malignant tumor that accounts for $11 \%$ of all retroperitoneal malignant tumors. The main therapy is resection, but clinical outcome following resection is poor if the coma regrows and invades adjacent organs and blood vessels, isolated iliac artery aneurysm is also a rare disease but presents a significant risk of life-threatening hemorrhage. In this article we reported a case of an acute rupture of an iliac artery aneurysm caused by invasion of retroperitoneal leiomyosarcoma. The patient was successfully treated by resection of the pelvic retroperitoneal leiomyosarcoma and reconstruction of iliac artery.
\end{abstract}

Volume 7 Issue 2 - 2017

\section{Xicheng Zhang, Zhaolei Chen, Yuan Sun, Yuanhu Jing, Miao Xu}

Department of Vascular Surgery, the Medical College of Yangzhou University, China

\begin{abstract}
Correspondence: Xicheng Zhang, Department of Vascular Surgery, the Medical College of Yangzhou University, Yangzhou, China,Email vasdoc@।26.com
\end{abstract}

Received: August 0I, 2017| Published: August 09, 2017

\section{Case report}

A 66-year-old female presented with a $>10$-year history of hypertension. She began to experienced dull pain in the lower abdomen and abdominal distension two days prior to admission. When she defecated six hours before admission, the pain suddenly aggravated and gradually spread to the whole abdomen, accompanied by dizziness, nausea, and vomiting, as well as symptoms of shock, including prefuse whole-body sweating and chills in all four limbs. Blood pressure was only $74 / 59 \mathrm{mmHg}$, she was semilucid with a pained expression, and limbs were wet and cold. Her stomach was distended and felt soft. The whole abdomen was sensitive to applied pressure, particularly the lower abdomen. Abdominal puncture was performed to draw a sample of non-condensable blood. Immediate emergency care included rapid fluid infusion and a blood transfusion. A CT scan revealed an abdominal enhancement consistent with expansion of the right iliac artery and there were masses in the soft tissues adjacent to the common iliac artery. There also appeared to be retroperitoneal hematomas, hence rupture of the aneurysm was suspected (Figure 1). Emergent exploratory laparotomy was performed under general anesthesia, and $\approx 3000 \mathrm{ml}$ of clotted blood was removed. There was still active bleeding at the site where the pelvic retroperitoneal rupture occurred. Pressure was applied to the site, hematocele was drawn completely. The abdominal aortic branch to the renal lower segment was quickly dissociated and controlled, after which active bleeding decreased significantly. The right iliac artery was expanded significantly to around $3.0 \mathrm{~cm}$ in diameter and dilation extended to the initial parts of the common and internal iliac artery. Inside the artery was a solid tumor about $5.0 \mathrm{~cm} \times 5.0 \mathrm{~cm} \times 4.0 \mathrm{~cm}$. The surface of the tumor was ulcerated and bled, its center was necrotized and liquefied and it appeared fish-flesh. The lower border was unclear, and the other extreme was close to the bifurcation of the right common iliac artery (Figure 2). The distal and proximal arteries to the right iliac aneurysm were re-clamped and finely dissociated from around the solid tumor.
The distant end of the aneurysm was found to be eroded by ulceration of the solid tumor, forming a laceration about $0.6 \mathrm{~cm}$ in diameter. The laceration was connected to the necrotized center of the solid tumor. The bottom of the solid tumor adhered to the pelvic wall. The solid tumor and the right iliac aneurysm were completely resected, and a prosthetic graft $(\mathrm{D}=8 \mathrm{~mm}$, Intervascular) were used to form an endto-end anastomosis of the common external iliac arteries (Figure 3). Postoperative pathology showed fusocellular sarcoma cells in a fascicular arrangement with high mitotic counts and cytoplasmic acidophilia, consistent with retroperitoneal leiomyosarcoma. Sections of the iliac artery expanded with the tumor and cholesterol crystals were observed in the vessel wall under a microscope. Nearby giant cells reacted with calcification. Postoperative vital signs were fairly stable and the patient recovered without complications. Adjuvant chemotherapy was given after surgery. Follow-up continued for 16 months, at which time CT detected local recurrence, but the patient refused another operation or chemotherapy and died 20months later from tumor metastasis.

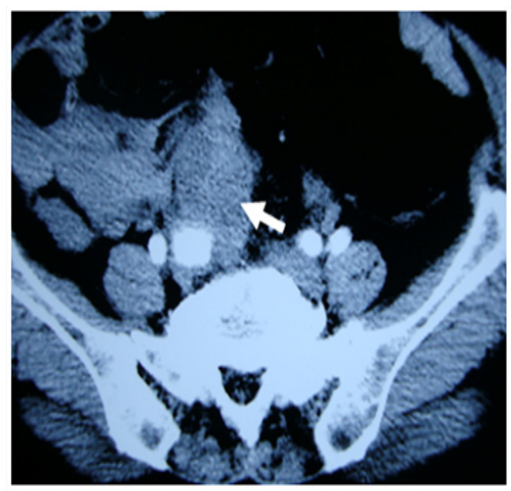

Figure I CT image showing the right iliac artery aneurysm. There are shadows of soft tissues near the aneurysm and retroperitoneal hematomas (white arrow). 


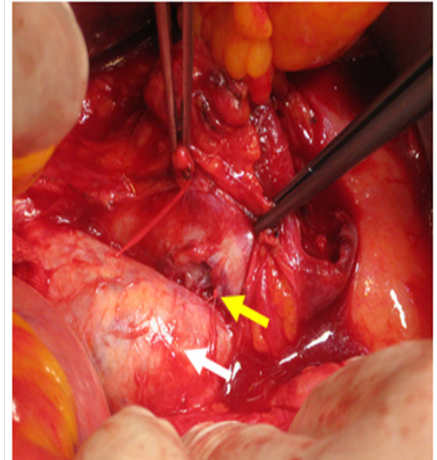

Figure 2 Intraoperative photograph revealing that the solid tumor had eroded the aneurysm wall (yellow arrow). The aneurysm ulcerated and bled from the necrotized and liquefied center of the solid tumor to the tumor surface. The white arrow points to the iliac artery aneurysm.

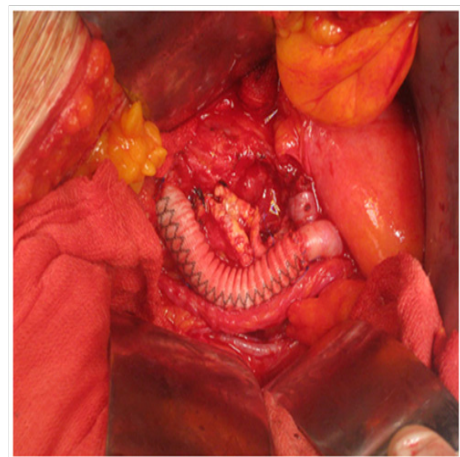

Figure 3 We resected the leiomyosarcoma and iliac artery aneurysm, and then repaired the artery using artificial blood vessels.

\section{Discussion}

Leiomyosarcoma is the third most common retroperitoneal sarcoma, and may occur in any retroperitoneal area or any area outside the pelvic peritoneum. This sarcoma is highly invasive with a 5-year survival rate of only $0 \% \sim 20 \%$. ${ }^{1,2}$ About two-thirds of retroperitoneal leiomyosarcoma cases occur in women, but the pathogenic factor is still unknown. The pathogenesis of retroperitoneal leiomyosarcoma is insidious without early specific symptoms. Therefore, the tumor tends to be large at presentation. Retroperitoneal leiomyosarcoma easily invades adjacent organs or large blood vessels, and may even erode the vessels, leading to rupture and hemorrhage. ${ }^{3}$ The first line therapy for retroperitoneal leiomyosarcoma is radical resection, and a tumor that involves large blood vessels may be resected with the vessels, followed by vascular remodeling. Chemotherapy is thought to have no significant effects on patient survival time, ${ }^{4}$ although a recent report concluded that some patients may experience beneficial effects on sunitinib. ${ }^{5}$ Isolated iliac artery aneurysm unaccompanied by abdominal aortic aneurysm is also a rare clinical event. ${ }^{6,7}$ Aneurysm is often insidious when in a deep location, and is difficult to find by physical examination when the coma is small or the patient obese. The greatest potential risk of aneurysm is vascular thrombosis of the limbs or rupture of comas. Once the comas rupture, the mortality will be high, reaching $30 \% \sim 50 \%{ }^{8,9}$ Treatment is challenging because most comas are located deep in the pelvic cavity where open operation can easily injure iliac veins, veins of the pelvic floor, or adjacent organs. In addition, most patients with aneurysm are elderly with many complications. Therefore, the operative risk is higher when the aneurysm ruptures. ${ }^{6}$ This case was very rare; in fact, to the best of our knowledge, this is the first report where retroperitoneal leiomyosarcoma had eroded the aneurysm on the right side and led to rupture. In such cases with signs of shock, the patient should be treated surgically as soon as possible. During the operation, the ends of the aneurysm were first blocked to control bleeding, and then the coma and associated blood vessels were separated and resected, followed by remodeling of the iliac artery. For those cases with little probability of resection because the (advanced) tumor has already invaded blood vessels and caused massive hemorrhage, transluminal therapy is an option to rapidly stop bleeding and save the patient. ${ }^{3}$ Local recurrence after surgery tends to be the main cause of death in patients with retroperitoneal leiomyosarcoma; hence, an improved resection rate will help improve prognosis. Close follow-up is vital after resection for early discovery of recurrent tumor foci and timely resection.

\section{Acknowledgements}

None.

\section{Conflict of interest}

The author declares no conflict of interest.

\section{References}

1. Royani B, Smith TA, Reith JD, et al. Retroperitoneal leiomyosarcomas unassociated with the gastrointestinal tract: a clincopathologic analysis of 17 cases. Mod Pathol. 1999;12(1):21-28.

2. Windham TC, Pisters PWT. Retroperitoneal sarcomas. Cancer Control. $2005 ; 12: 36-43$

3. Stambo G, Valentin M, Kerr TM, et al. Endovascular treatment of an acutely ruptured abdominal aorta from tumor invasion by unresectable retroperitoneal leiomyosarcoma. Ann Vasc Surg. 2008;22:568-570.

4. Mendenhall WH, Zlotecki RA, Hochwald SN, et al. Retroperitoneal soft tissue sarcoma. Cancer. 2005;104:669-675.

5. Mahmood ST, Agresta S, Vigil C, et al. Phase II study of sunitinib malate, a multitargeted tyrosine kinase inhibitor in patients with relapsed or refractory soft tissue sarcomas. Focus on 3 prevalent histologies: Leiomyosarcoma, liposarcoma, and malignant fibrous histiocytoma. Int J Cancer. 2011;129(8):1963-1969.

6. Patel NV, Long GW, Cheema ZF, et al. Open vs. endovascular repair of isolated iliac artery aneurysms: A 12-year experience. J Vasc Surg. 2009;49(5):1147-1153.

7. Boules TN, Selzer F, Stanziale SF, et al. Endovascular management of isolated iliac artery aneurysms. J Vasc Surg. 2006;44(1):29-37.

8. Richardson JW, Greenfield LJ. Natural history and management of iliac aneurysms. J Vasc Surg. 1988;8(2):165-171.

9. Sandhu RS, Pipinos II. Isolated iliac artery aneurysms. Semin Vasc Surg. 2005;18(4):209-215. 